LA-UR-01-5906

Approved for public release; distribution is unlimited.

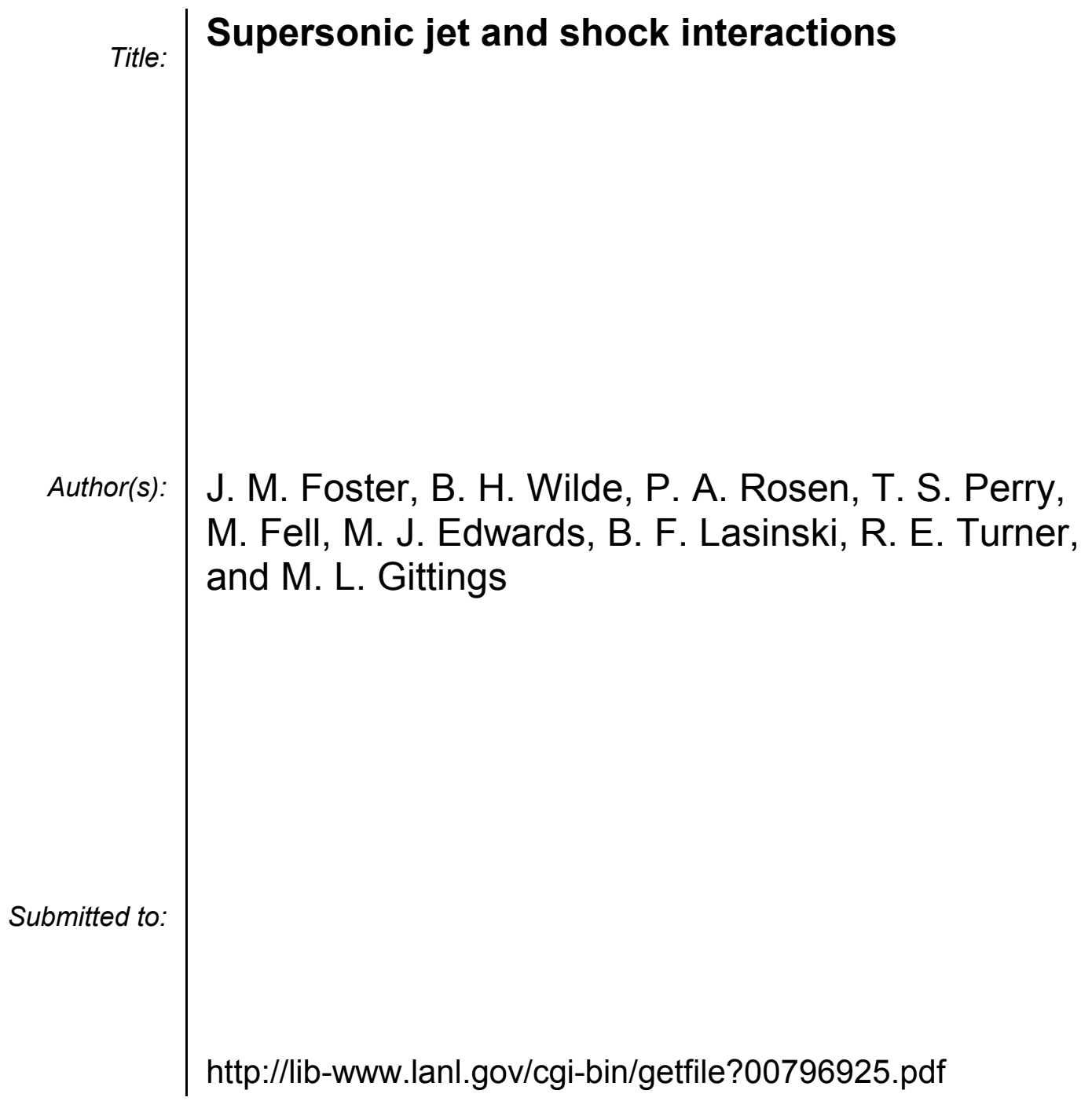

Los Alamos National Laboratory, an affirmative action/equal opportunity employer, is operated by the University of California for the U.S. Department of Energy under contract W-7405-ENG-36. By acceptance of this article, the publisher recognizes that the U.S. Government retains a nonexclusive, royaltyfree license to publish or reproduce the published form of this contribution, or to allow others to do so, for U.S. Government purposes. Los Alamos National Laboratory requests that the publisher identify this article as work performed under the auspices of the U.S. Department of Energy. Los Alamos National Laboratory strongly supports academic freedom and a researcher's right to publish; as an institution, however, the Laboratory does not endorse the viewpoint of a publication or guarantee its technical correctness. 


\title{
Supersonic jet and shock interactions ${ }^{a)}$
}

\author{
J. M. Foster, ${ }^{1, b)}$ B. H. Wilde, ${ }^{2}$ P. A. Rosen, ${ }^{1}$ T. S. Perry, ${ }^{3}$ M. Fell, ${ }^{1}$ M. J. Edwards, ${ }^{3}$ \\ B. F. Lasinski, ${ }^{3}$ R. E. Turner, ${ }^{3}$ and M. L. Gittings ${ }^{2,4}$ \\ ${ }^{1}$ AWE Aldermaston, Reading, RG7 4PR, United Kingdom \\ ${ }^{2}$ Los Alamos National Laboratory, Los Alamos, New Mexico 87544 \\ ${ }^{3}$ Lawrence Livermore National Laboratory, Livermore, California 94551 \\ ${ }^{4}$ Science Applications International Corporation, San Diego, California 92121
}

(Received 31 October 2001; accepted 20 February 2002)

\begin{abstract}
Supersonic fluid flow and the interaction of strong shock waves to produce jets of material are ubiquitous features of inertial confinement fusion (ICF), astrophysics, and other fields of high energy-density science. The availability of large laser systems provides an opportunity to investigate such hydrodynamic systems in the laboratory, and to test their modeling by radiation hydrocodes. We describe experiments to investigate the propagation of a structured shock front within a radiation-driven target assembly, the formation of a supersonic jet of material, and the subsequent interaction of this jet with an ambient medium in which a second, ablatively driven shock wave is propagating. The density distribution within the jet, the Kelvin-Helmholz roll-up at the tip of the jet, and the jet's interaction with the counterpropagating shock are investigated by x-ray backlighting. The experiments were designed and modeled using radiation hydrocodes developed by Los Alamos National Laboratory, AWE, and Lawrence Livermore National Laboratory. The same hydrocodes are being used to model a large number of other ICF and high energy-density physics experiments. Excellent agreement between the different simulations and the experimental data is obtained, but only when the full geometry of the experiment, including both laser-heated hohlraum targets (driving the jet and counter-propagating shock), is included. The experiments were carried out at the University of Rochester's Omega laser [J. M. Soures et al., Phys. Plasmas 3, 2108 (1996)].
\end{abstract} [DOI: $10.1063 / 1.1468858]$

\section{INTRODUCTION}

The supersonic flow of fluids, and the formation and interaction of strong shock waves, are ubiquitous features of the nonlinear hydrodynamics of inertial-confinement fusion (ICF), astrophysics, and related fields of high energy-density science. Shock waves that traverse ICF capsule defects ${ }^{1,2}$ (hemispherical joints, surface bumps, and other imperfections) may lead to the formation of jets of material that initiate mixing of ablator and fuel and thus degrade yield. In the astrophysical context, highly collimated jets ${ }^{3}$ originate from active galactic nuclei, young stellar objects, and planetary nebulae. Herbig-Haro objects ${ }^{4-6}$ are understood to be visible as a result of emission features arising from shocks during the interaction between proto-stellar jets and the interstellar medium; their structure has been modeled ${ }^{7-9}$ and is sensitive (among other parameters) to the Mach number of the jet, the density contrast between jet and ambient medium, and radiative losses. In the late stages of a star's evolution, jets arising as a result of magneto-rotational stellar collapse ${ }^{10}$ may play an important role in the explosion of some types of supernovae. ${ }^{11}$

Laboratory studies of supersonic jets ${ }^{12-14}$ have until recently been limited to relatively low pressure and Mach number, and to temperatures for which radiation transport is not an important mechanism. Consequently, detailed under-

\footnotetext{
${ }^{a)}$ Paper QI2 1, Bull. Am. Phys. Soc. 46, 248 (2001).

${ }^{b}$ Invited speaker. Electronic mail: john.foster@awe.co.uk
}

standing of many phenomena for high Mach-number and radiative jets has been founded solely in numerical simulation using radiation hydrodynamic codes employing a variety of different algorithms for approximate solution of the fundamental nonlinear hydrodynamic equations. The availability of high-power lasers, such as Omega, now provides a means of studying supersonic fluid flow and shock interactions under conditions formerly unavailable in the laboratory, and of experimentally testing the numerical algorithms implemented in different radiation hydrocodes. Thus, laser drive has been used to investigate the radiative collapse of high Mach-number jets, ${ }^{15,16}$ although so far only under conditions where the jet density exceeds that of the ambient medium by many orders of magnitude. The general problem of scaling from these and other laboratory experiments to the astrophysical context has been studied in detail by Ryutov and co-workers. ${ }^{17,18}$

In the work reported here, the Omega laser ${ }^{19}$ was used (in indirect drive mode) to launch a spatially structured converging shock front into a composite target assembly, leading to the formation of a well-collimated, high Mach-number jet. We have investigated the interaction of this jet with an ambient medium which is either static, or in which a second, near-planar shock is propagating toward the jet.

We report the first comprehensive laboratory study of the processes leading to formation of the jet and its interaction with the surrounding material. The density distribution within the jet, the jet shock and the bow shock in the ambient 


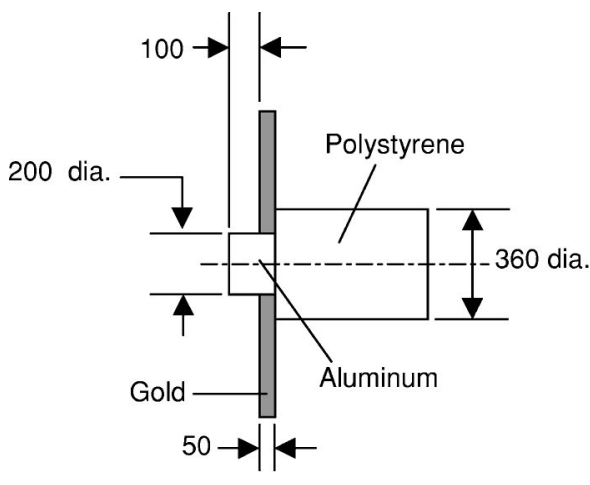

(a)

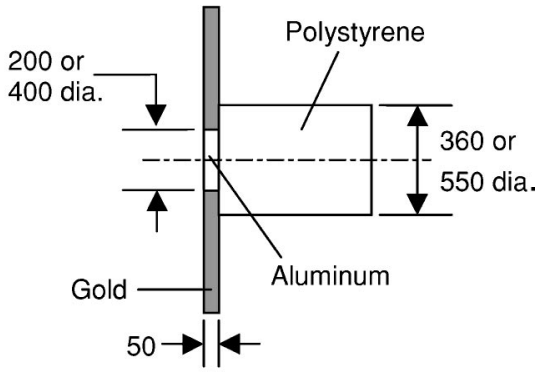

(b)

FIG. 1. (a) The experimental package for the aluminum-jet experiment consists of a cylindrical aluminum "pin" surrounded by a gold "washer," and an adjacent cylindrical polystyrene block. (b) Experimental package for the planar-geometry experiment. Dimensions are in microns.

medium, the Kelvin-Helmholz roll-up of the tip of the jet, and the jet's subsequent interaction with a second, counterpropagating shock are diagnosed by x-ray backlighting. The objective of the experiment is to provide detailed quantitative data for comparison with the results of radiation hydro- code simulations employing different calculational models. We compare the experimental data with the results of simulations employing adaptive-mesh-refinement Eulerian (AMR), fixed-mesh Eulerian, and arbitrary Lagrangian Eulerian (ALE) calculational schemes. These calculations were carried out using the hydrocodes RAGE, PETRA, and CALE, developed, respectively, by Los Alamos National Laboratory (LANL), AWE, and Lawrence Livermore National Laboratory (LLNL).

\section{EXPERIMENTAL DETAILS}

\section{A. Experimental package, hohlraum, and radiography}

\section{Supersonic-jet experiment}

The basic experimental package [Fig. 1(a)] consists of a cylindrical aluminum "pin," surrounded by a gold "washer." $\mathrm{X}$-ray driven ablation of the exposed end and side surfaces of the pin results in the inward propagation of strong (100 Mbar) shock waves. Interaction of these shocks near axis leads to the subsequent formation of a jet of material at the outer (not driven) end of the pin. The particular choice of dimensions for the pin and washer affects the form and degree of collimation of the jet. We use a 200- $\mu$ m-diam pin, set within a $50-\mu \mathrm{m}$-thick, $1000-\mu \mathrm{m}$-diam washer. In most cases, the pin projects $100 \mu \mathrm{m}$ from the driven face of the washer, and is flush with the rear face. However we have also used experimental packages in which the pin is of different length, and projects from the washer by either 50 or $200 \mu \mathrm{m}$ (see Sec. IV). The jet, formed as a result of shock interaction within the pin, propagates into a cylindrical $(360-\mu \mathrm{m}$-diam, typically $500-\mu \mathrm{m}$-length) solid polystyrene block placed adjacent to the outer face of the pin. Figure 2 shows the density distribution, and the positions of material interfaces, taken from a radiation hydrodynamic simulation of the experiment using the LANL hydrocode RAGE, to illustrate the processes


(a)


Shock in $\mathrm{Al}$ Bow shock in $\mathrm{CH}$
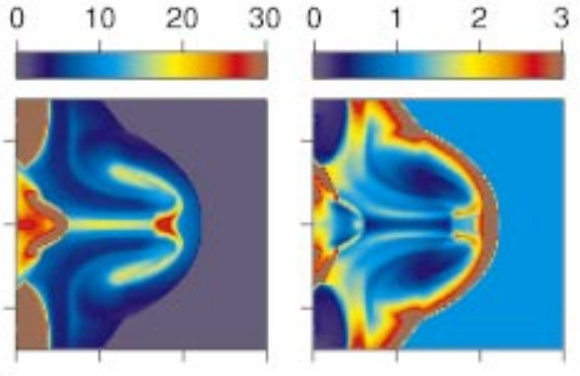

FIG. 2. (Color) (a) Density distribution during shock transit within the aluminum pin, showing processes leading to formation of the jet. Ablatively driven shocks from the (cylindrical) side and (planar) end of the pin interact in an annular, Mach-stem-like structure (identified by arrows) which converges on axis where the jet is formed. The solid black line shows the location of the interface between aluminum and the surrounding materials. (b) The fully developed aluminum jet at $t=8 \mathrm{~ns}$. The flow velocity of material within the jet $\left(u_{\text {jet }}\right)$ is decelerated by a shock located near the head of the jet. The contact discontinuity separating jet and ambient materials propagates supersonically in the ambient material (polystyrene), leading to the formation of a bow shock. These features are evident in the temperature and density distributions (center and right). In both (a) and (b), the distance between tick marks is $100 \mu \mathrm{m}$. 
leading to formation of the jet, and its subsequent interaction with the ambient medium (polystyrene). This and other simulations are discussed in further detail in Sec. IV B.

Radiation drive is generated using a $1600-\mu \mathrm{m}$-diam, $1200-\mu \mathrm{m}$-length (internal dimensions) cylindrical gold hohlraum ${ }^{20}$ target with a single $1200-\mu \mathrm{m}$-diam laser entry hole. The hohlraum wall thickness is typically $50 \mu \mathrm{m}$. The experimental package is mounted over an $800-\mu \mathrm{m}$-diam hole in the end wall of the hohlraum, opposite the laser entry hole. This axisymmetric configuration enables us to model the entire (hohlraum plus experimental package) assembly using two-dimensional radiation hyrocodes. The hohlraum is heated using 12 beams of the Omega laser with a total energy of $6 \mathrm{~kJ}$ in a $1 \mathrm{~ns}$ duration, constant-power laser pulse of $0.35 \mu \mathrm{m}$ wavelength. The resulting peak radiation drive temperature lies in the range 190-200 eV. The 12 laser spots are equispaced in azimuth around the cylindrical sidewall of the hohlraum, to optimize drive uniformity at the experimental package.

The density distribution within the jet, and the bow shock in the surrounding polystyrene, are measured using $\mathrm{x}$-ray backlighting radiography along two (mutually orthogonal) lines of sight, perpendicular to the axis of symmetry of the hohlraum and experimental package. Two x-ray backlighting laser targets (one for each line of sight) are used, positioned $4 \mathrm{~mm}$ from the experimental package. Each target consists of a 3-mm-square, $25-\mu \mathrm{m}$-thickness foil of titanium illuminated with six laser beams (400-600 ps pulse duration, $0.35 \mu \mathrm{m}$ wavelength), incident from both sides of the target, smoothed using random phase plates and superimposed in an $800-\mu \mathrm{m}$-diam spot. The backlighter spectrum is dominated by the resonance line emission of helium-like titanium at $4.75 \mathrm{keV}$.

$\mathrm{X}$-ray backlit images of the jet are recorded on each line of sight by an eight-times magnification pinhole camera. Each camera incorporates a 16-hole array of $10-\mu \mathrm{m}$-diam pinholes, and the 16 synchronous images are recorded on Kodak DEF x-ray film. Temporal resolution is determined by the duration of the $\mathrm{x}$-ray backlighting pulse, and each camera incorporates a $15-\mu \mathrm{m}$-thickness titanium-foil filter to select the resonance line radiation of the $\mathrm{x}$-ray backlighting target. The time delays (relative to the hohlraum drive) of the backlighter beams are adjusted so that the images recorded on the two cameras are either synchronous, or sequential, in time.

\section{Planar-shock experiment}

In addition to investigating the hydrodynamics of jet formation and propagation, our principal aim (as a stringent test of radiation hydrocode modeling) has been to study the interaction of the jet with a counterpropagating, quasiplanar shock in the ambient medium surrounding the jet. For this purpose, we have developed a target assembly in which a thin, disk-shaped, aluminum ablator drives a near-planar shock through the polystyrene cylinder. Studies of this target assembly have been valuable in their own right: the hydrodynamics tests modeling of the hohlraum radiation drive, but also reveals details of a late-time, pressure drive that results from filling of the hohlraum by ablated material from the
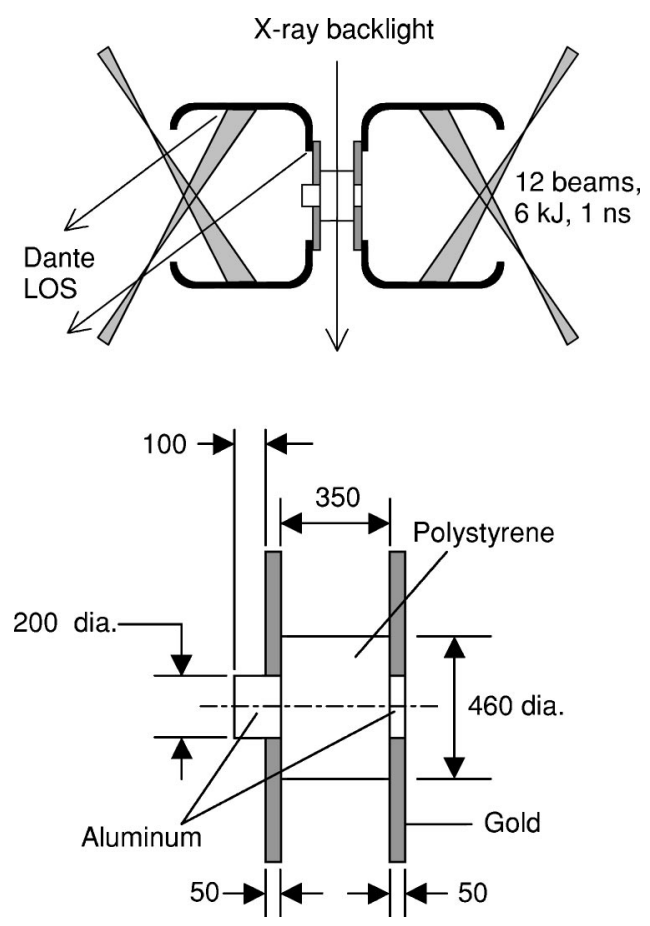

FIG. 3. The counterpropagating jet and shock experiment is driven by two hohlraums on a common axis. Dimensions are in microns.

hohlraum's wall. Knowledge of this late-time pressure drive, and its accurate modeling, are important in the interpretation of experiments driven by hohlraum targets. ${ }^{21,22}$ The target assembly consists of a 200- or 400- $\mu$ m-diam aluminum disk, set (flush with both faces) within a 1000- $\mu$ m-diam gold washer [Fig. 1(b)]. Both disk and washer are $50 \mu \mathrm{m}$ thick. Again, a solid polystyrene cylinder (360 or $550 \mu \mathrm{m}$ diameter, typically $500 \mu \mathrm{m}$ length) is positioned adjacent to the outer face of the ablator and washer. This experimental package is mounted over an 800- $\mu \mathrm{m}$-diam hole in the end wall of the hohlraum, exactly as for the experimental package used in the jet experiments. Other details of the experiment and target diagnostics are also as for the jet experiment.

\section{Counterpropagating jet and shock}

In the case of a counterpropagating jet and shock, we use two experimental packages at opposite ends of a common central polystyrene component (Fig. 3). In this case, the 200$\mu \mathrm{m}$-diam pin projects $100 \mu \mathrm{m}$ from the face of its surrounding washer, and the counterpropagating shock is driven by a $200-\mu \mathrm{m}$-diam aluminum pusher. The central polystyrene component is $460 \mu \mathrm{m}$ diameter and $350 \mu \mathrm{m}$ long. The whole experimental assembly is driven by two laser-heated hohlraum targets, mounted on a common axis. All other details of diagnostics and radiation drive are as for the jet experiment.

\section{Data reduction}

Summation of the 16 synchronous images provided by the open-shutter pinhole camera provides a means of improving the signal-to-noise ratio of the data, and smoothing spatial variations of backlighter intensity. The image data on film are digitized using a PDS scanning microdensitometer, 
and film density pixel values are converted to exposure units. The 16 images are summed, using a correlation-function method to ensure correct image alignment. Because the backlighting source lies in a plane $4 \mathrm{~mm}$ from that of the experimental package, parallax results in a somewhat different relative position of experimental package and backlighting target in each image: the image summation procedure therefore inherently provides some smoothing of backlighter spatial variations. In cases where the periphery of the image includes a significant area of unattenuated backlighter, we remove spatial variations of backlighter intensity by using a polynomial fitting procedure to infer the unattenuated backlighter intensity which underlies the image of the experimental package. We divide the backlit image data by the inferred, spatially varying unattenuated backlighter intensity, to obtain a map of $\mathrm{x}$-ray transmission through the experimental package. It is this measured transmission data that we compare with postprocessed hydrocode calculations that simulate the experimental radiographs. As a test of the fidelity of this technique, measurement of the x-ray transmission of the undisturbed polystyrene cylinder (ahead of the bow shock) is typically within $\leqslant 2 \%$ of calculation.

Figures 7, 12 and 13 (see the following) show examples of the radiographic data from the three categories of experiment, together with simulated radiographs from different hydrocodes. The data clearly show the aluminum jet penetrating the surrounding polystyrene material, the resulting bowshock in the polystyrene, and Kelvin-Helmholz roll-ups at the tip of the jet. These features form the basis for quantitative comparison of the experimental data with hydrodynamic simulations.

\section{B. Hohlraum radiation-drive characterization}

In addition to $\mathrm{x}$-ray backlighting measurements of jet formation and interaction, we have made measurements of the hohlraum radiation drive for comparison with our detailed radiation-hydrodynamic modeling.

We use a 10-channel array of filtered vacuum x-ray diodes ${ }^{23,24}$ (known as "Dante") to record time-resolved emission from the hohlraum laser-entrance hole, in the 0.1$2.0 \mathrm{keV}$ region of the $\mathrm{x}$-ray spectrum. The Dante diagnostic views through the laser-entry hole at an angle of $37.4^{\circ}$ relative to the hohlraum axis. With this line of sight, both the directly heated hohlraum wall (that is, some of the laser hot spots) and the indirectly heated wall contribute to the x-ray emission recorded by Dante. ${ }^{25}$ Radiation configuration-factor calculations indicate that the relative contribution of each to the measured emission spectrum is rather close to the relative contributions made by hot-spot and wall emission to the radiation drive experienced at the experimental package. In the absence of laser entry-hole closure, the Dante diagnostic therefore provides an approximate indication of the radiation-drive spectrum and "temperature" (the equivalent brightness temperature of the measured spectrum) at the experimental package.

Although the Dante data alone at early time provide a close approximation to the radiation drive experienced by the experiment, we prefer to consider the measured $\mathrm{x}$-ray emis-

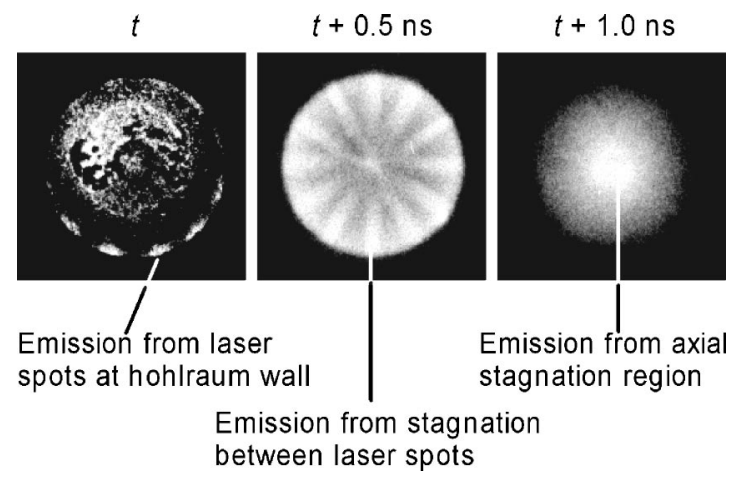

FIG. 4. Images from an $x$-ray framing camera viewing axially through the hohlraum laser-entry hole. The images are separated by $0.5 \mathrm{~ns}$ intervals in time, and the first image occurs close to the rise of the 1 ns laser pulse.

sion spectrum from the laser-entrance hole in the context of our detailed, integrated model of the hohlraum.

We construct a detailed radiation-hydrocode model of the hohlraum and experimental package, including a treatment of laser-energy deposition at the hohlraum wall. Furthermore, we postprocess this simulation, to calculate the $\mathrm{X}$-ray emission spectrum (that is, the specific intensity) emitted through the laser-entrance hole in the direction of Dante. It is the comparison of this spectrum with experimental data, together with comparison of the observed and calculated hydrodynamics (aluminum ablator and shock trajectories) for the planar-geometry experiment, that is the basis for testing our hohlraum modeling.

In the following we describe further details of the hohlraum modeling, and the radiation-drive prescription that we use in hydrocode simulations (RAGE, PETRA, and CALE) that do not include a treatment of laser-energy deposition.

We also describe in the following how the hydrodynamics of the experiment are affected, at late time, by the inward motion and consequent tamping provided by the hohlraum walls. We have been able to model successfully the gross features of this effect by including the hohlraum walls in our hydrodynamic model, but very accurate modeling is difficult because of the three-dimensional nature of coronal plasma expansion from the discrete laser hot spots at the hohlraum wall. We have investigated the expansion and interaction of the laser hot-spot plasmas by using a multichannel, timegated x-ray pinhole camera, ${ }^{26}$ viewing axially through the laser entry hole. The camera was filtered by a $125-\mu \mathrm{m}-$ thickness beryllium filter, and thus responds to $\mathrm{x}$ rays of photon energy $>1 \mathrm{keV}$. Figure 4 shows three sequential images from this camera, at $0.5 \mathrm{~ns}$ intervals in time. Emission from the neighborhood of the individual laser hot spots is followed by emission from stagnation regions (appearing rather like spokes of a wheel) between the hot spots. These features are followed a further $0.5 \mathrm{~ns}$ later by bright emission from an axial stagnation region.

\section{HOHLRAUM MODELING AND RADIATION-DRIVE PRESCRIPTION}

\section{A. Hohlraum modeling}

Our approach to modeling the jet experiments is threefold. We start by developing a detailed model for the 
radiation-temperature drive provided by the hohlraum target. For this, we use principally the AWE NYM hydrocode which includes laser ray-trace and laser energy deposition packages, and the necessary nonlocal-thermodynamic equilibrium (NLTE) physics. Second, we test this hohlraum model against measurements of hohlraum x-ray emission (recorded using the Dante diagnostic) and the observed hydrodynamics of the planar-geometry experiments. Finally, we apply the tested hohlraum model to define a radiation-temperature drive for use in detailed simulations of the jet hydrodynamics using the RAGE, PETRA, and CALE hydrocodes.

In this section, we discuss the detailed hohlraum model and its comparison with the experimental $\mathrm{x}$-ray emission data. We discuss the hydrodynamics of the planar geometry experiments in the first part of Sec. IV.

We have used the $\mathrm{NYM}^{27}$ Lagrangian radiation hydrocode for the detailed hohlraum modeling. The hohlraum (including the $200-\mu \mathrm{m}$-diam planar-geometry experimental package) is modeled in two-dimensions, with laser light incident in a hollow cone, illuminating an annular ring at the hohlraum wall. Laser energy absorption is modeled as inverse bremsstrahlung in the coronal plasma, with in addition a $30 \%$ deposition of energy at the critical surface. Radiation transport is treated by multigroup Monte Carlo photonics. ${ }^{28}$ NLTE physics in the laser absorption region is treated by the ZEUS package, which provides in-line time-dependent NLTE modeling by solution of rate equations for average-ion populations in the screened-hydrogenic approximation. This NLTE treatment is applied in selected cells of the simulation, including and bordering the region of laser transport and energy deposition; elsewhere, tabular LTE opacities are used, calculated off-line using the IMP ${ }^{29}$ opacity code. Equationof-state is also input in tabular form, provided by off-line simulation. The Lagrangian zoning is sufficiently fine to resolve x-ray radiative heating and re-emission at the hohlraum wall, and ablation of the aluminum experimental package. The void within the hohlraum is treated as low density $\left(10^{-3}\right.$ $\mathrm{g} \mathrm{cm}^{-3}$ ) hydrocarbon $(\mathrm{CH})$.

The NYM calculation proceeds until $2 \mathrm{~ns}$ after the beginning of the laser pulse. After this time, the calculational time-step becomes impracticably small, and we transfer the simulation to the AWE Eulerian hydrocode PETRA. ${ }^{30,31}$ In this phase of the calculation, no further energy is input from an external source and the code tracks the further evolution of the hohlraum and experimental package. Radiation transport is treated by single-group (gray) diffusion. Ion, electron, and radiation temperatures are assumed equal (1-T model). The (fixed) Eulerian mesh is coarser than the zoning used in parts of the Lagrangian (NYM) simulations, although it is sufficiently fine near the axis to resolve stagnation of the inwardly expanding hohlraum walls and their interaction with the experimental package.

\section{B. Simulated and measured Dante data}

For comparison with the experimental hohlraum characterization data, we postprocess the NYM and PETRA simulations, to calculate the $\mathrm{x}$-ray emission spectrum in the direction of the Dante diagnostic (that is, the spatial integral, over

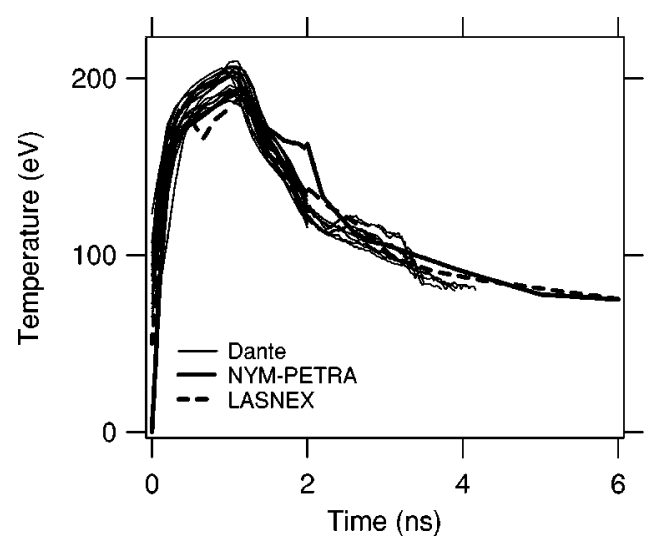

FIG. 5. Apparent brightness temperature of the hohlraum wall. The experimental (Dante diagnostic) data are from three different experimental series and do not incorporate hole-closure corrections. In the case of the simulations, a brightness temperature has been defined by treating the simulated emission spectra from the postprocessed NYM-PETRA and LASNEX simulations in the same way as the experimental data, and the effects of holeclosure are thus implicitly included.

the source, of the specific intensity). This ray-tracing postprocessing tracks rays through all parts of the laser entry hole, and therefore explicitly includes the effects on the emitted spectrum of laser entry-hole closure at late time.

Comparison with the experimental data is possible at several levels, although it is convenient (particularly in the context of a radiation-drive temperature prescription) to consider the effective brightness temperature of the hohlraum wall.

In defining a brightness temperature from the experimental Dante diagnostic data it is impractical to account for laser entry-hole closure. Instead, the measured spectrum is assumed to originate from a source whose area is equal to that of the 1200- $\mu \mathrm{m}$-diam (original size, before closure) laser-entry hole. The x-ray emission flux (integral over space and spectrum) inferred by this method is equated to that from an idealized Planckian source, to obtain an effective source brightness temperature for the area of hohlraum wall viewed by Dante. For purposes of comparison only, we have treated our simulated hohlraum emission spectra (the results of postprocessing the hydrocode simulations) in exactly the same way. By ignoring the effects of hole closure, and treating the simulated emission spectrum in exactly the same way as the experimental data, we define an effective brightness temperature that is directly comparable with the experimental data (but nevertheless somewhat smaller than reality).

Figure 5 compares our experimental hohlraum brightness temperature data with the postprocessed NYM and PETRA simulations. In addition to the NYM-PETRA simulations, we also show in Fig. 5 the results of a LASNEX ${ }^{32}$ simulation of the hohlraum. In the case of LASNEX, the total outward flux from the laser entry hole, as a function of time, has been extracted from the simulation. Again, for comparison with experiment, we define the brightness temperature of an equivalent Planckian source whose area is assumed to be that of the original laser-entry hole. 


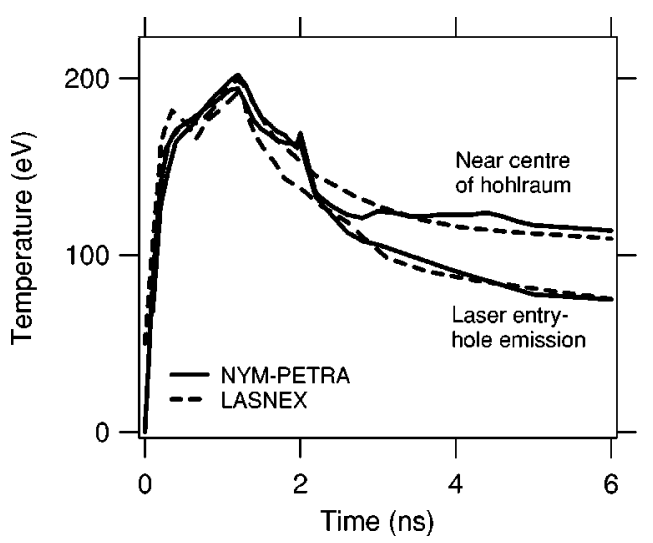

FIG. 6. Radiation temperature within the hohlraum in the detailed NYMPETRA model (solid curve). The discontinuity at $t=2 \mathrm{~ns}$ is associated with linking from the Lagrangian (NYM) to the Eulerian (PETRA) model. Similar data from LASNEX are shown by the dashed curve. Note that the hohlraum radiation temperature at late time is higher than the brightness temperature of emission from the laser entry hole, because of hole closure.

\section{Radiation drive prescription}

For use in the RAGE, PETRA, and CALE simulations of the jet experiments, we require a temperature-drive prescription. We obtain this from the detailed NYM-PETRA hohlraum model described previously, by extracting the radiation temperature from a location within the hohlraum bordering the experimental package. Figure 6 shows this hohlraum radiation-temperature, taken from both the NYM-PETRA detailed hohlraum model and also the LASNEX simulation.

\section{HYDRODYNAMIC MODELING AND COMPARISONS WITH EXPERIMENTAL DATA}

\section{A. General comments and hydrocode descriptions}

We have modeled the experiments with radiation hydrocodes employing adaptive-mesh-refinement (AMR), fixedmesh Eulerian, and arbitrary Lagrangian Eulerian (ALE) calculational schemes. These calculations were carried out using the hydrocodes RAGE, PETRA, and CALE and used a temperature-input drive prescription. Additionally, we have modeled the planar-geometry experiments using NYM coupled to PETRA, and laser-light input, as described in Sec. III. The radiation-temperature drive prescription for the RAGE, PETRA, and CALE simulations was that described in Sec. III.

The design of the experiments used mainly the LANL hydrocode RAGE, although many simulations were also done with the AWE NYM and PETRA codes. The LLNL CALE hydrocode was used primarily for postshot analysis of the jet-shock interaction experiments.

RAGE (radiation adaptive grid Eulerian) is a multidimensional, multimaterial Eulerian radiation-hydrodynamics code developed by Los Alamos National Laboratory and Science Applications International (SAIC). ${ }^{33}$ RAGE uses a continuous (in time and space) adaptive-mesh-refinement (CAMR) algorithm to follow interfaces and shocks, and gradients of physical quantities such as material densities and temperatures. At each cycle, the code automatically determines whether to subdivide or recombine Eulerian cells. The user also has the option to de-zone (that is, reduce the resolution of the mesh) as a function of time, space, or material. Adjacent square cells may differ by only one level of resolution, that is, by a factor of 2 in cell size. Although the code does not have interface tracking or reconstruction, it easily follows contact discontinuities with fine zoning at the material interfaces. This CAMR method speeds calculations by as much as two orders of magnitude over straight Eulerian methods. RAGE uses a second-order-accurate Godunov hydrodynamics scheme similar to the Eulerian scheme of Colella. ${ }^{34}$ Mixed cells are assumed to be in pressure and temperature equilibrium, with separate material and radiation temperatures. Radiation transport is approximated with implicit gray flux-limited diffusion. The code has been validated with analytical test problems and many shock-tube, laser and pulsed-power experiments. ${ }^{1,2,35,36}$

The physics incorporated in the (Lagrangian) NYM and (Eulerian) PETRA calculations is described in Sec. III.

CALE is an ALE (arbitrary Lagrangian Eulerian) hydrodynamics code developed by LLNL. ${ }^{37}$ Calculations for the present experiment were carried out in $(r, z)$ geometry in pure Eulerian mode with a minimum spatial resolution of 2.5 $\mu \mathrm{m}$. Calculations performed at $1 \mu \mathrm{m}$ resolution or in ALE mode gave similar results, which differed only in minor details. The laser-hohlraum interactions were not directly modeled. Instead, a temperature drive was imposed at the boundaries of the problem which remained fixed in time. As a result, the backpressure from the hohlraum that begins to affect the experimental data late in time is not included (although this effect is not significant in the case of the jetshock interaction experiments modeled by CALE). Radiation transport was modeled in the gray diffusion approximation and opacity and equation-of-state were generated off-line and input in tabular form.

\section{B. Jet formation, scaling, and turbulence}

Figure 2(a), taken from a RAGE simulation of the experiment, shows the temporal evolution of material densities and interfaces during the formation of the aluminum jet and its subsequent emergence into the polystyrene receptacle. The ablation of the side and end faces of the cylindrical aluminum pin by the hohlraum radiation sends strong shocks into the aluminum and these shocks interact in an annular Mach-stem-like structure (identified by arrows in the figure), lying on the surface of a cone and converging toward the axis. The jet arises from the convergence on axis of this conical Mach-stem shock front and its formation may thus be loosely analogous to convergent flows in the astrophysical context proposed by Cantó et al. as a possible mechanism for jet production. ${ }^{38,39}$ As the jet enters the solid polystyrene cylinder the flow is supersonic with respect to the ambient medium; the directed flow within the jet is decelerated by a shock internal to the beam and located near the head of the jet (the decelerated material at the head of the jet forms the mushroom-headed shape characteristic of Kelvin-Helmholz instability); and the contact discontinuity separating the jet 
and ambient materials propagates supersonically in the ambient material, leading to the formation of a bow shock [Fig. 2(b)].

The maximum density attained at early time inside the developing jet is approximately $15 \mathrm{~g} \mathrm{~cm}^{-3}$ and the internal pressure exceeds $100 \mathrm{Mb}$. The material is in equilibrium with the radiation with a maximum temperature of $50 \mathrm{eV}$ in the jet and consequently the jet does not cool significantly via radiation losses. Nevertheless, the simulations require modeling of radiation transport to obtain the correct ablation of the aluminum and the hohlraum walls. Temperatures and densities in the fully developed jet are rather lower, as discussed in the following. Zoning studies, with both RAGE and PETRA, determined that minimum cell sizes of about $1 \mu \mathrm{m}$ were required to obtain convergence of features within the experimental resolution of $12 \mu \mathrm{m}$.

We have carried out experiments using aluminum pins with a diameter of $200 \mu \mathrm{m}$ and lengths (exposed from the surrounding washer) of 50, 100, and $200 \mu \mathrm{m}$. The $100 \mu \mathrm{m}$ length pin (reported here) forms a jet with well-developed Kelvin-Helmholtz roll-ups at the mushroom head. A $50 \mu \mathrm{m}$ length pin barely forms a jet and its mushroom is not as well developed. The internal shock interactions also create a broad stem with wings. A $200 \mu \mathrm{m}$ length pin forms a jet with a significantly smaller mushroom head and stem diameter than the 100- $\mu$ m-length pin. These features observed in experiment are reproduced by our modeling of the experiment, but are not reported in detail here.

The morphology of the jets created in our experiment shows many points of similarity with numerical models of astrophysical supersonic jets, and the question arises of the extent to which the experiment can be scaled to systems of perhaps vastly different physical size. Such scaling has been studied in detail by Ryutov et al., ${ }^{17,18}$ who show that if dissipative processes are negligible, then scaling transformations exist that enable the hydrodynamic evolution of the laboratory system to be mapped onto that of another system at different physical size, providing that certain dimensionless scaling parameters are common to both systems.

The requirement for negligible dissipative processes demands that viscosity and thermal conduction are negligible; it is quantified through the Reynolds number, $\operatorname{Re}=u_{\text {jet }} r_{\text {jet }} / \nu$ and the Peclet number, $\mathrm{Pe}=u_{\text {jet }} r_{\text {jet }} / \kappa$, where $\nu$ and $\kappa$ are the kinematic viscosity and thermal diffusivity, respectively, and $u_{\text {jet }}$ and $r_{\text {jet }}$ are the flow velocity within the jet and the jet radius. Systems in which $\mathrm{Re} \gg 1$ and $\mathrm{Pe} \gg 1$ are those in which inertial forces and advection of heat dominate viscous drag and thermal conduction.

Using Spitzer's ${ }^{40}$ expressions for the viscosity and thermal conductivity, we obtain the following for the kinematic viscosity and thermal diffusivity:

$$
\nu\left(\mathrm{cm}^{2} \mathrm{~s}^{-1}\right)=3.20 \times 10^{-5} \frac{T(\mathrm{eV})^{5 / 2} A^{1 / 2}}{Z^{4} \ln \Lambda \rho\left(\mathrm{g} \mathrm{cm}^{-3}\right)},
$$

and

$$
\kappa\left(\mathrm{cm}^{2} \mathrm{~s}^{-1}\right)=3.30 \times 10^{-3} \frac{T(\mathrm{eV})^{5 / 2} A}{Z(Z+1) \ln \Lambda \rho\left(\mathrm{g} \mathrm{cm}^{-3}\right)} .
$$

TABLE I. Physical conditions in the fully developed jet at $t=9 \mathrm{~ns}$, and corresponding dimensionless scaling numbers.

\begin{tabular}{lccc}
\hline \hline Quantity & Symbol & Value & \\
\hline Temperature & $T$ & 20 & $\mathrm{eV}$ \\
Density & $\rho_{\text {jet }}$ & 0.3 & $\mathrm{~g} \mathrm{~cm}^{-3}$ \\
Mean ionization & $Z$ & 4 & \\
Coulomb logarithm & $\ln \Lambda$ & 2 & \\
Fluid velocity in jet & $u_{\text {jet }}$ & $5 \times 10^{6}$ & $\mathrm{~cm} \mathrm{~s}^{-1}$ \\
Jet radius & $r_{\text {jet }}$ & 10 & $\mu \mathrm{m}$ \\
Density ratio & $\rho_{\text {jet }} / \rho_{\text {ambient }}$ & 0.3 & \\
& & & \\
Sound speed in jet & $c_{s}=\sqrt{(Z+1) k T / m_{i}}$ & $2 \times 10^{6}$ & $\mathrm{~cm} \mathrm{~s}^{-1}$ \\
Internal Mach number & $M=u_{\text {jet }} / c_{s}$ & 3 & \\
Thermal diffusivity & $\kappa$ & 10 & $\mathrm{~cm}^{2} \mathrm{~s}^{-1}$ \\
Peclet number & $\mathrm{Pe}=u_{\text {jet }} r_{\text {jet }} / \kappa$ & 500 & \\
Kinematic viscosity & $\nu$ & $2 \times 10^{-3}$ & $\mathrm{~cm}^{2} \mathrm{~s}^{-1}$ \\
Reynolds number & $\operatorname{Re}=u_{\text {jet }} r_{\text {jet }} / \nu$ & $2.5 \times 10^{6}$ & \\
Radiative cooling time & $\tau_{\text {rad }}$ & 7 & $\mathrm{~ns}$ \\
Cooling parameter & $\chi=u_{\text {jet }} \tau_{\text {rad }} / r_{\text {jet }}$ & 20 & \\
\hline \hline
\end{tabular}

For the fully developed jet in our experiment (at, say, $t=9 \mathrm{~ns})$ we have $u_{\text {jet }}=50 \mu \mathrm{m} \mathrm{ns}^{-1}$ and $r_{\text {jet }}=10 \mu \mathrm{m}$. Corresponding values of the mean ionization, $Z$, the Coulomb logarithm, $\ln \Lambda$, and $\mathrm{Re}$ and Pe are listed in Table I.

The properties of the jet also depend upon certain macroscopic dimensionless scaling numbers, ${ }^{7,8}$ specifically the Mach number, $M=u_{\text {jet }} / c_{s}$, the density contrast ratio, $\rho_{\text {jet }} / \rho_{\text {ambient }}$, and the radiative cooling parameter, $\chi$ $=u_{\text {jet }} \tau_{\text {rad }} / r_{\text {jet }}$, where $\tau_{\text {rad }}$ is a typical time scale for radiative cooling. We may place a lower limit on the radiative cooling time by assuming that the jet is a Planckian emitter. It follows that

$$
\tau_{\text {rad }}=\frac{[\text { thermal energy per unit length }]}{[\text { radiative loss per unit length }]},
$$

and

$$
\tau_{\mathrm{rad}} \geqslant \frac{\pi r_{\mathrm{jet}}^{2} \frac{3}{2}(Z+1) k T n_{\mathrm{i}}}{2 \pi r_{\mathrm{jet}} \sigma T^{4}} .
$$

Typical values for the macroscopic dimensionless scaling numbers are listed in Table I.

It is interesting to note that the hydrodynamic development of the jet in our experiment (Fig. 12, in the following) shows a number of similarities with the low Mach number jets considered by Norman et al. ${ }^{7}$ Specifically, Norman's Fig. 10(b) shows the development of a Kelvin-Helmholz roll-up for a jet with $M=1.5$ and $\rho_{\text {jet }} / \rho_{\text {ambient }}=1.0$, conditions similar to our own $M=3$ and $\rho_{\text {jet }} / \rho_{\text {ambient }}=0.3$. Other cases considered by Norman et al. $\left(M=6,3,1.5\right.$ and $\rho_{\text {jet }} / \rho_{\text {ambient }}$ $=0.1$ ) show somewhat different hydrodynamics, including the development of a "cocoon" of backflowing gas that may be accessible to future, appropriately scaled laboratory experiments.

Norman et al. ${ }^{7}$ note that the qualitative structure of a supersonic jet and its interaction with the surrounding medium is quite different from a subsonic jet. In the high Reynolds-number, subsonic case, the surrounding medium is entrained into the jet through turbulent mixing, ${ }^{41}$ whereas in 



FIG. 7. Simulated and experimental radiographs of the 400- $\mu$ m-diam planar-geometry experiment at $t=15 \mathrm{~ns}$ : (a) RAGE simulations without (top) and with (bottom) inclusion of the hohlraum driver; (b) experimental data.

the supersonic case the flow remains largely parallel and well collimated at the slip discontinuity separating the jet and ambient materials, and shock structures internal to the beam are developed. ${ }^{42,43}$ This may in part explain the highly collimated appearance of the jet in our experiment, in the region of maximum flow velocity before the decelerating shock internal to the jet. However, it is also important to consider the time scale necessary for the establishment of near "steadystate" turbulence. Hinze ${ }^{44}$ discusses the early time development of free jets, and notes that distinct, separate vortices, generated by Kelvin-Helmholz instability along the boundary of the jet, only evolve into turbulent flow late in time. Furthermore, recent laser-driven laboratory jet experiments by Raga et al., ${ }^{45}$ although subsonic, show very similar structure (a well-collimated jet, with a distinct mushroom roll-up) to our own experiment, although they were conducted in the high Reynolds-number regime in which we would expect turbulent flow to develop late in time. It is therefore interesting to calculate approximately the expected amplitude of turbulent mixing at the boundary of the jet in our own experiment, in the region of maximum flow velocity: The KelvinHelmholz growth rate ${ }^{46}$ for wave number $k$ is of order $\gamma$ $=k u\left[\rho_{1} \rho_{2} /\left(\rho_{1}+\rho_{2}\right)^{2}\right]^{0.5}$. Assuming, say, ten growth times before saturation, we can roughly calculate when turbulent growth of scale equal to the spatial resolution of our experiment (approximately $10 \mu \mathrm{m}$ ) will appear. Taking $u=50$ $\mu \mathrm{mns}^{-1}, \lambda=20 \mu \mathrm{m}, k=2 \pi / \lambda=3.1 \times 10^{3} \mathrm{~cm}^{-1}$, and $\rho_{1} / \rho_{2}$ $=0.3$, we find $\gamma=6.5 \times 10^{9} \mathrm{~s}^{-1}$. Over a period of ten growth times $\left(1.5 \mathrm{~ns}\right.$, for $\left.\gamma=6.5 \times 10^{9} \mathrm{~s}^{-1}\right)$ our jet propagates a dis-

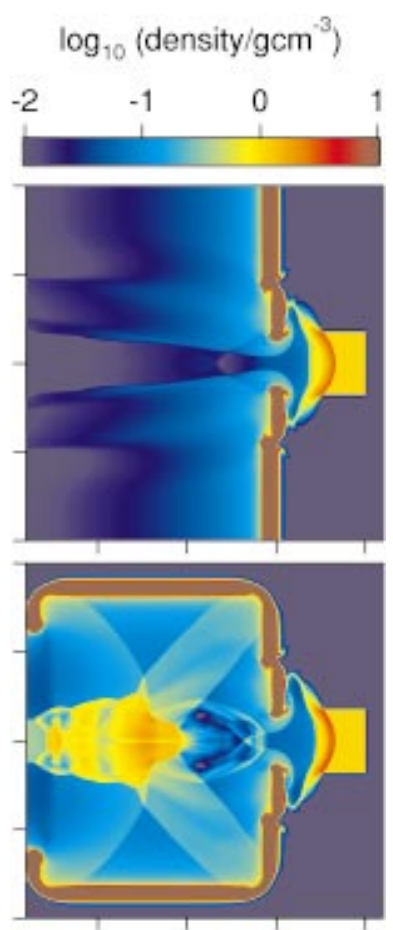

FIG. 8. (Color) Density distribution in RAGE simulations of the $200-\mu \mathrm{m}-$ diam planar-geometry experiment at $t=10 \mathrm{~ns}$, without (top) and with (bottom) inclusion of the hohlraum wall. The distance between tick marks is 500 $\mu \mathrm{m}$.

tance of $75 \mu \mathrm{m}$. It is interesting to note in passing that this approximately calculated turbulent growth of $10 \mu \mathrm{m}$ over a distance of $75 \mu \mathrm{m}$ corresponds to an expansion angle of $\tan ^{-1}(10 / 75)=8^{\circ}$-roughly that observed in subsonic turbulent jet experiments. ${ }^{41}$ A distance of $75 \mu \mathrm{m}$ is comparable to the length of the fully developed jet in our experiment, so turbulent mixing may be marginally below the diagnostic resolution limit. We conclude that although the high Mach number of our jet may in part be responsible for its high degree of collimation, the absence of turbulent mixing may also be because sufficient time is simply not available for turbulence to develop, even though the Reynolds number is very high.

\section{Planar-geometry experiments, and further tests of the hohlraum model}

The planar-geometry experiments (effectively pins of zero exposed length, and with diameters of 200 and $400 \mu \mathrm{m}$ ) were designed to produce a quasiplanar shock and to investigate the effects of hohlraum filling (that is, the additional drive pressure created by tamping of ablated material by the hohlraum, and the effects of the inward-moving hohlraum walls in amplifying this tamping effect and ultimately forming a jet of gold following on-axis stagnation of the hohlraum wall).

Figure 7(a) shows synthetic x-ray backlighter images from (postprocessed) RAGE simulations of the 400- $\mu \mathrm{m}-$ diam planar target at $15 \mathrm{~ns}$ after onset of the radiation drive. The bottom half of Fig. 7(a) is taken from a RAGE simulation which included the gold hohlraum driver; the top half 
used precisely the same radiation-drive prescription but did not include the hohlraum. Although the leading shock in the polystyrene block is at effectively the same position in the two simulations, the aluminum pusher has a very different shape and has advanced to a different position in space. Figure 7(b) shows the experimental data. The simulation that includes the hohlraum driver shows best match to the experimental data, both in the position of the aluminumpolystyrene interface and in the density distribution in the neighborhood of the ablation surface of the aluminum. Figure 8 shows the density distribution within the hohlraum, or in the neighborhood of the ablated aluminum disk, in two simulations of a 200- $\mu \mathrm{m}$-diam planar experiment, again without and with inclusion of the hohlraum. Inspection of the two simulations reveals that the ablation and inward motion of the hohlraum walls tamps the ablation of the aluminum disk and creates late-time pressures in the ablated aluminum that are several times greater than those in the simulation without the hohlraum. Clearly, at sufficiently late time, modeling of the experiment cannot treat the hydrodynamics of the experimental package in isolation, but must also consider its hydrodynamic coupling with the hohlraum driver.

This point is well illustrated by the displacement-time trajectories of the aluminum-polystyrene interface, and the shock in polystyrene, in the with- and without-hohlraum simulations. Figures 9 and 10 show the experimentally measured interface and shock positions, as functions of time, for
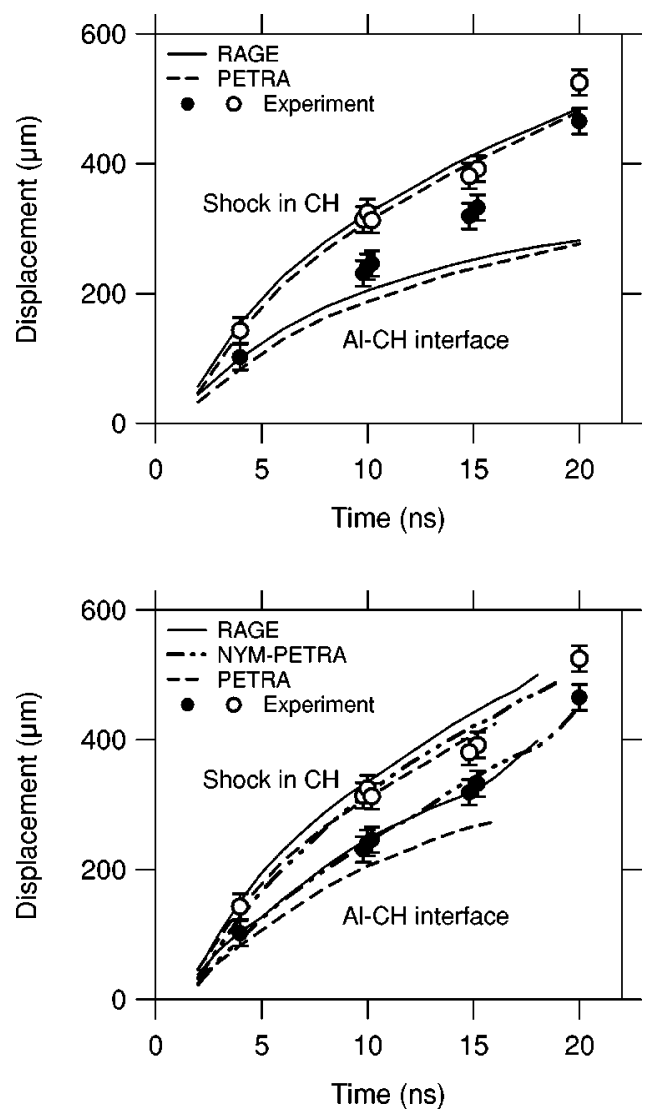

FIG. 9. Interface and shock displacement for the 200- $\mu$ m-diam planargeometry experiment, without (top) and with (bottom) inclusion of the hohlraum in the simulation.
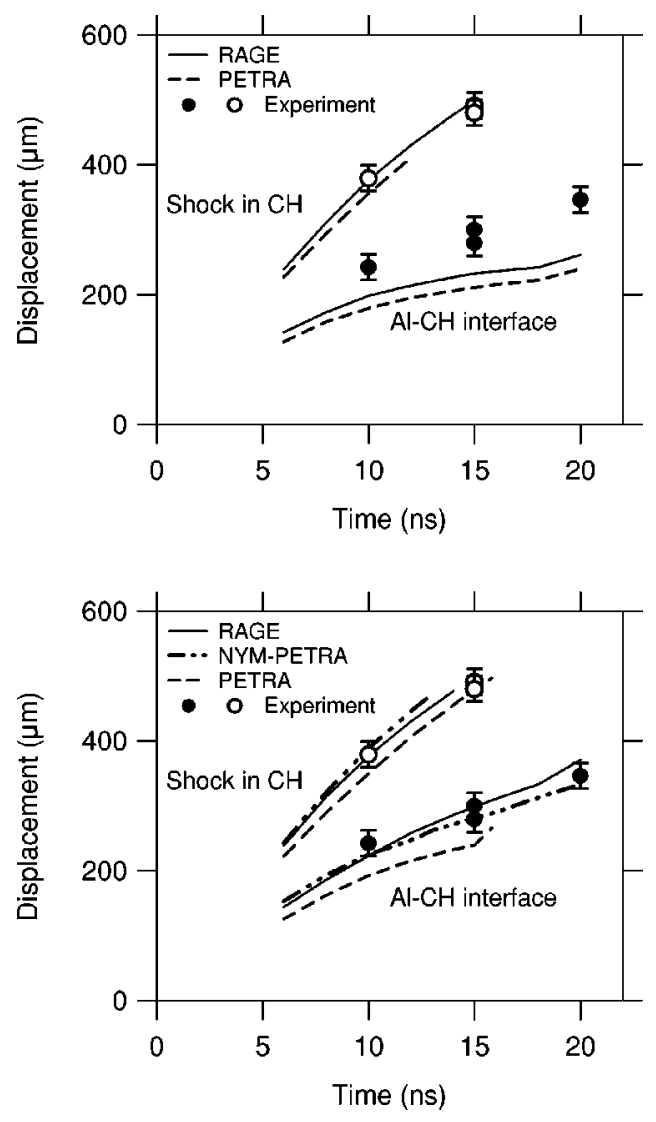

FIG. 10. Interface and shock displacement for the 400- $\mu$ m-diam planargeometry experiment, without (top) and with (bottom) inclusion of the hohlraum in the simulation.

planar-geometry experiments using 200- and 400- $\mu$ m-diam planar aluminum ablators. Also shown are the results of RAGE, NYM-PETRA, and PETRA calculations. Both figures indicate that a significantly better match to experiment is obtained when the hohlraum is included in the simulations. Hohlraum filling begins to have a noticeable effect after about $7 \mathrm{~ns}$ and becomes significant after 15-17 ns when the RAGE simulations start to show a gold jet resulting from ablation of the hohlraum walls and the subsequent on-axis stagnation of the ablated gold. Our two-dimensional cylindrically symmetric RAGE simulations tend to overestimate this late-time gold jetting due to the perfect symmetry assumed in the calculations, and at late time it is sufficient to penetrate the aluminum ablator. As shown in Fig. 4, the laser-spot ablation of the gold walls is actually highly threedimensional. This breaks the two-dimensional symmetry and the experimental data show rather less hohlraum-wall jetting than we find in the hydrocode simulations. Nevertheless, experiments do indicate some gold jetting, originating in the hohlraum, on the ablation side of the aluminum at very late times ( $20 \mathrm{~ns})$. These late-time gold jets are quite sensitive to the zoning of the hohlraum and changes in the driving temperature. They are less evident in the NYM-PETRA simulations of the hohlraum, as these start from a laser light input prescription and thus include a better approximation of laserspot ablation effects (albeit in two-dimensional approximation) than RAGE or PETRA alone. Further exploration of 



FIG. 11. Jet-tip and bow-shock displacement for the $100-\mu \mathrm{m}-\mathrm{long}$ pin experiment, without (top) and with (bottom) inclusion of hohlraum in the simulation.

this effect awaits the inclusion of additional physics in our new three-dimensional ASCI codes (such as RAGE).

\section{Jet experiments}

Given the excellent agreement of simulations with experimental data in the planar geometry, we now turn to the data from the jet experiment. Figure 11 shows the displacement (on axis) as a function of time, of the tip of the aluminum jet, and the bow shock in the polystyrene block ahead of the jet. These experimental data for the jet-tip and bow-shock positions are compared with the results of RAGE and PETRA simulations, again with and without inclusion of the hohlraum. Comparison of the with- and without-hohlraum simulations indicates that hohlraum filling does not significantly effect the displacement of the jet tip until approximately 9 ns after the onset of the radiation drive. Figure 12 shows experimental radiographs of the jet at 10 and $15 \mathrm{~ns}$ after onset of radiation drive together with RAGE simulations at a similar point in the development of the hydrodynamics (same displacement of the jet and bow shock). We note that although the shock and interface positions are not as sensitive to the hohlraum filling as in the planar-geometry experiment, the width of the mushroom stem is significantly broadened when the hohlraum is included in the modeling. Again, the with-hohlraum model is in better agreement with

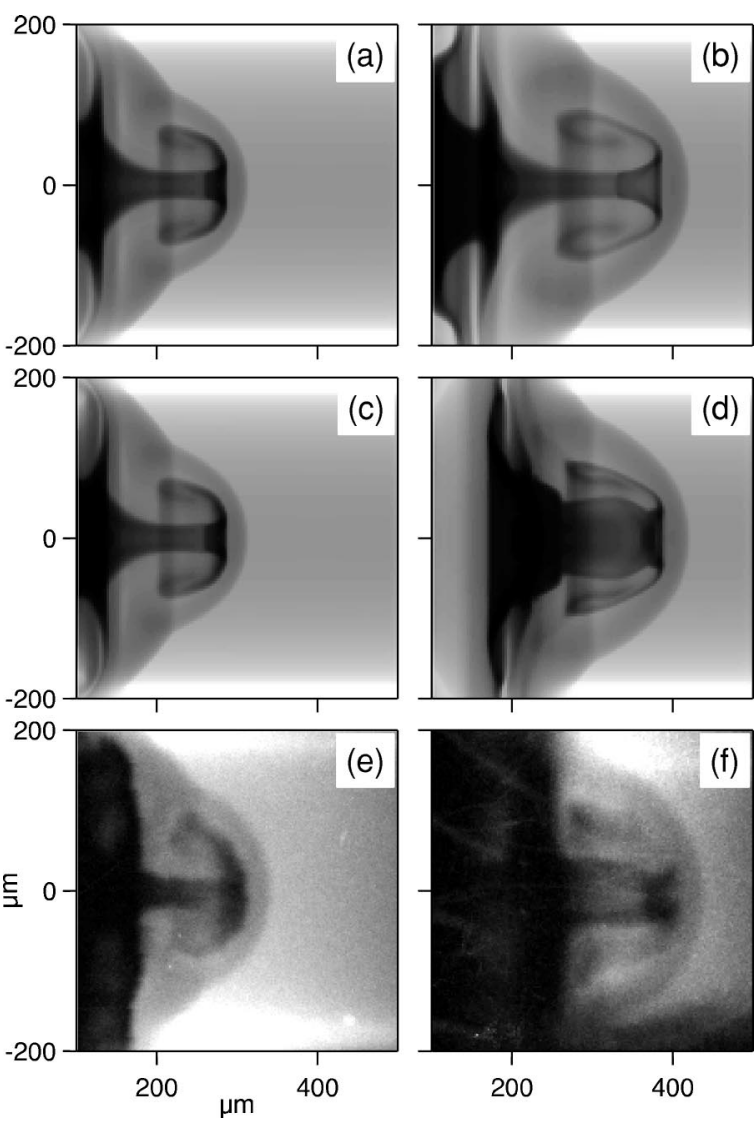

FIG. 12. Simulated and experimental radiographs of the $100-\mu \mathrm{m}-$ long pin experiment: (a), (b) RAGE simulations at $t=8$ and $12 \mathrm{~ns}$, in which the hohlraum is not included in the modeling; (c), (d) RAGE simulations at $t$ $=8$ and $12 \mathrm{~ns}$, in which the hohlraum is included in the modeling; (e), (f) experimental data at $t=10$ and $15 \mathrm{~ns}$, at a similar stage in the development of the jet motion.

experimental data. In the case of the with-hohlraum simulation, the calculation also shows that the jet develops a hollow core at late time, as revealed by experiment.

RAGE simulations for the $100-\mu$ m-length pins are in agreement with the experimental data at early times (4-10 ns) but tend to show greater displacement at late times. This is in contrast to RAGE and NYM-PETRA simulations of the 200- and 400- $\mu \mathrm{m}$-diam planar-geometry experiments which are in excellent agreement with the data at all times. Using a peak temperature profile approximately $14 \mathrm{eV}$ lower (within the limits of uncertainty of the Dante data) RAGE simulations agree rather better with the data. However, the difference between experiment and simulation may also be due to the development of three-dimensional features as a result of asymmetric drive or surface perturbations. The experimental data for the jet in Figs. 12(e) and 12(f) certainly show some three-dimensional asymmetries, and the energy thus lost would tend to slow the jet. We plan to carry out threedimensional calculations to investigate this conjecture.

\section{E. Jet and counterpropagating shock experiments}

In the case of the jet-shock interaction experiments, we limit the duration of the experiment to within $8 \mathrm{~ns}$ of the onset of radiation drive, and thus eliminate some of the un- 

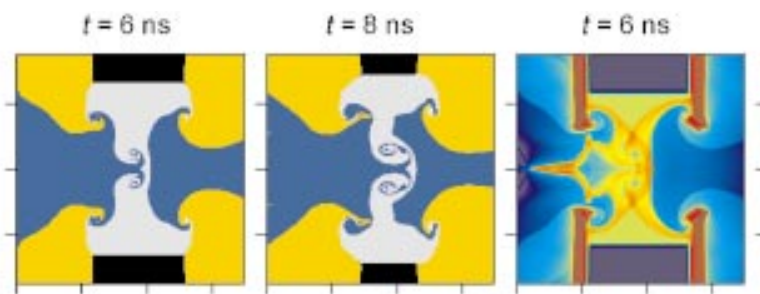

(a)


(b)

certainties associated with hohlraum filling. Figure 13(a) shows material interfaces and density distributions taken from a RAGE simulation of the jet-shock interaction; experimental $\mathrm{x}$-ray backlighting radiographs of the interaction, in comparison with RAGE, PETRA, and CALE simulations at 4, 6, and 8 ns are shown in Fig. 13(b). Each simulation reproduces the essential features of the hydrodynamic flow. To obtain a more quantitative comparison of experiment and modeling, we consider contours of equal backlighter transmission.

Figure 14 compares transmission contours (absolute $\mathrm{x}$-ray backlighter transmission $=0.3$ and 0.4 ) that locate the



FIG. 14. (Color) Contours of $x$-ray transmission from the experimental data (black curve), and the RAGE (red), PETRA (blue), and CALE (green) simulations. The transmission values have been chosen to locate a position close to that of the bow shock in the polystyrene block. The distance between tick marks is $100 \mu \mathrm{m}$.
FIG. 13. (Color) (a) Material interfaces and density distribution in a RAGE simulation of the counterpropagating jet and shock experiment. The distance between tick marks is 200 $\mu \mathrm{m}$. (b) X-ray backlighting radiographs from the jet-shock interaction experiment, compared with simulations using the RAGE, PETRA, and CALE hydrocodes, at $t=4,6$, and 8 ns. The hohlraum wall thickness is 21 $\mu \mathrm{m}$ in the experiment and RAGE simulation, $50 \mu \mathrm{m}$ in the PETRA and CALE simulations. The gray-scale has been chosen to bring out particular features of the interaction, and does not represent the full range of $\mathrm{x}$-ray transmission; the distance between tick marks is $100 \mu \mathrm{m}$. 
bow shock in the cylindrical polystyrene block at times of 4 and $6 \mathrm{~ns}$ after onset of the radiation drive. The agreement of the data with the three calculations is within the $12 \mu \mathrm{m}$ spatial resolution of the experiment. Figure 15 compares transmission contours (transmission $=0.1$ and 0.05 ) that lie close to the interface between the aluminum and the polystyrene plastic. Again the comparison with the data and between the three radiation-hydrodynamic codes is within the experimental resolution. These comparisons are absolute in time, space, and transmission values. Except for postshot modifications that were necessary to account for the actual laser pulseshape used, and difference of hohlraum-wall thickness (50 $\mu \mathrm{m}$ planned, $21 \mu \mathrm{m}$ as shot), the RAGE calculations were preshot predictions.

Figures 13-15 show x-ray transmission data at 4, 6, and $8 \mathrm{~ns}$. We have also obtained additional titanium-backlighter data at $4.5,5,5.5,7,7.5$, and $9 \mathrm{~ns}$, and data using a (somewhat more penetrating) vanadium backlighter at similar times. All of the data agree with the simulations of the three codes to within experimental uncertainties.

\section{CONCLUSIONS}

We have reported the results of a series of laser-driven experiments to investigate the production of collimated dense-plasma jets following the interaction of strong shocks, and the subsequent interaction of these jets with a further ablatively driven, counterpropagating shock.

Because they exercise many aspects of two-dimensional hydrodynamics and radiation transport, these experiments have provided an excellent vehicle for hydrocode benchmarking: both verification through the comparison of different simulations of the same experiment, and validation through comparison of the results of these calculational models with the experimental data. Simulation of the experiment using a wide range of calculational tools using both traditional and modern hybrid calculational techniques has been very successful in reproducing the experimental data, but only when the full geometry of the experiment, including the laser-heated hohlraum driver(s) is included. The jetting phenomena addressed by these simulations are similar to those encountered in many instances of hydrodynamic instability, and the well-resolved, quantitative nature of the present experimental data provides a rigorous test, of wide relevance, of different modeling techniques. In performing simulations of the experiment, some of the issues addressed are general in nature, while others are specific to a particular calculational model or technique. Probably the most basic issue relates to spatial resolution, and the trade-off between calculational speed and fidelity. Both the type of calculational mesh, and the mesh resolution, have an important bearing on the speed and accuracy of the simulations. The experiment provides a valuable test of mesh adaptation strategies in calculational schemes such as AMR, as well as a benchmark against which to test simpler traditional hydrodynamic models at different resolution (different cell sizes). In particular, the convergence of the ablation-driven shock in the pin provides a benchmark against which to test the validity of modeling in the difficult near-axis region. The experimental data are radiographic density measurements, and comparison with hydrocode simulation is obtained through comparison with synthetic radiographs obtained by postprocessing of the hydrocode simulations. The experiment has therefore also exercised data visualization techniques of importance in the wider context of many proposed high energy-density physics experiments.

The need to include the hohlraum driver in the simulations results from hydrodynamic coupling between the ablated material from the experimental package and the inward-expanding hohlraum wall. Although this tamping effect is relatively unimportant at early time, it is significant on the 10-20 ns time scale and should ideally be accounted for in other experiments of similar duration using the same hohlraum driver.

\section{ACKNOWLEDGMENTS}

The authors warmly thank Bruce Remington, Mike Dunne, and Peter Graham for their advice and several helpful discussions, and gratefully acknowledge the technical expertise of Steve Compton, Vern Rikow, and Sharon Alvarez, and the staffs of the Omega laser facility and the AWE and LLNL target-fabrication groups.

${ }^{1}$ S. R. Goldman, S. E. Caldwell, M. D. Wilke, D. C. Wilson, C. W. Barnes, W. W. Hsing, N. D. Delamater, G. T. Schappert, J. W. Groves, E. L. Lindman, J. M. Wallace, R. P. Weaver, A. M. Dunne, M. J. Edwards, P. Graham, and B. R. Thomas, Phys. Plasmas 6, 3327 (1999).

${ }^{2}$ S. R. Goldman, C. W. Barnes, S. E. Caldwell, D. C. Wilson, S. H. Batha, J. W. Grove, M. L. Gittings, W. W. Hsing, R. J. Kares, K. A. Klare, G. A. Kyrala, R. W. Margevicious, R. P. Weaver, M. D. Wilke, A. M. Dunne, M.

J. Edwards, P. Graham, and B. R. Thomas, Phys. Plasmas 7, 2007 (2000).

${ }^{3}$ P. J. Witta, Nature (London) 355, 499 (1992).

${ }^{4}$ B. Reipurth and S. Heathcote, Astron. Astrophys. 246, 511 (1991).

${ }^{5}$ R. Mundt, Can. J. Phys. 64, 407 (1986).

${ }^{6}$ S. Heathcote, J. A. Morse, P. Hartigan, B. Reipurth, R. D. Schwartz, J. Bally, and J. M. Stone, Astron. J. 112, 1141 (1996).

${ }^{7}$ M. L. Norman, L. Smarr, K.-H. A. Winkler, and M. D. Smith, Astron. Astrophys. 113, 285 (1982).

${ }^{8}$ J. M. Blondin, A. Königl, and B. A. Fryxell, Astrophys. J. Lett. 337, L37 (1989).

${ }^{9}$ J. M. Stone and M. L. Norman, Astrophys. J. 413, 198 (1993).

${ }^{10}$ J. M. LeBlanc and J. R. Wilson, Astrophys. J. 161, 541 (1970).

${ }^{11}$ A. M. Khokhlov, P. A. Höflich, E. S. Oran, J. C. Wheeler, L. Wang, and A. Yu. Chtchelkanova, Astrophys. J. Lett. 524, L107 (1999).

${ }^{12}$ R. Ladenburg, C. C. Van Voorhis, and J. Winckler, Phys. Rev. 76, 62 (1949).

${ }^{13}$ P. J. Finley, J. Fluid Mech. 26, 337 (1966).

${ }^{14}$ J. S. Shang, J. Hayes, K. Wurtzler, and W. Strang, AIAA J. 39, 1159 (2001).

${ }^{15}$ D. R. Farley, K. G. Estabrook, S. G. Glendinning, S. H. Glenzer, B. A. Remington, K. Shigemori, J. M. Stone, R. J. Wallace, G. B. Zimmerman, and J. A. Harte, Phys. Rev. Lett. 83, 1982 (1999).

${ }^{16}$ K. Shigemori, R. Kodama, D. R. Farley, T. Koase, K. G. Estabrook, B. A. Remington, D. D. Ryutov, Y. Ochi, H. Azechi, J. M. Stone, and N. Turner, Phys. Rev. E 62, 8838 (2000).

${ }^{17}$ D. D. Ryutov, R. P. Drake, J. Kane, E. Liang, B. A. Remington, and W. M. Wood-Vasey, Astrophys. J. 518, 821 (1999).

${ }^{18}$ D. D. Ryutov, B. A. Remington, H. F. Robey, and R. P. Drake, Phys. Plasmas 8, 1804 (2001).

${ }^{19}$ J. Soures, R. L. McCrory, C. P. Verdon et al., Phys. Plasmas 3, 2108 (1996).

${ }^{20}$ J. Lindl, Phys. Plasmas 2, 3933 (1995).

${ }^{21}$ B. H. Wilde, M. L. Gittings, T. S. Perry, J. M. Foster, P. A. Rosen, M. Fell, and J. A. Cobble, Bull. Am. Phys. Soc. 44, 39 (1999).

${ }^{22}$ O. A. Hurricane, S. G. Glendinning, B. A. Remington, R. P. Drake, and K. K. Dannenberg, Phys. Plasmas 8, 2609 (2001). 
${ }^{23}$ H. N. Kornblum, R. L. Kauffman, and J. A. Smith, Rev. Sci. Instrum. 57, 2179 (1986).

${ }^{24}$ R. L. Kauffman, H. N. Kornblum, D. W. Phillion, B. F. Lasinsi, L. J. Suter, A. R. Thiessen, R. J. Wallace, and F. Ze, Rev. Sci. Instrum. 66, 678 (1995).

${ }^{25}$ C. Decker, R. E. Turner, O. L. Landen, L. J. Suter, P. Amendt, H. N. Kornblum, B. A. Hammel, T. J. Murphy, R. W. Wallace, N. D. Dalamater, P. Gobby, A. A. Hauer, G. R. Magelssen, J. A. Oertel, J. Knauer, F. J. Marshall, D. Bradley, W. Seka, and J. M. Soures, Phys. Rev. Lett. 79, 1491 (1997).

${ }^{26}$ K. S. Budil, T. S. Perry, P. M. Bell, J. D. Hares, P. L. Miller, T. A. Peyser, R. Wallace, H. Louis, and D. E. Smith, Rev. Sci. Instrum. 67, 485 (1996).

${ }^{27}$ P. D. Roberts, AWE Aldermaston (unpublished); P. D. Roberts, S. J. Rose, P. C. Thompson, and R. J. Wright, J. Phys. D 13, 1957 (1980).

${ }^{28}$ J. A. Fleck and J. D. Cummings, J. Comput. Phys. 8, 313 (1971).

${ }^{29}$ S. J. Rose, J. Phys. B 25, 1667 (1992).

${ }^{30}$ D. L. Youngs, in Numerical Methods for Fluid Mechanics, edited by K. W. Morton and M. J. Baines (Academic, London, 1982).

${ }^{31}$ D. L. Youngs, Physica D 12, 32 (1984).

${ }^{32}$ G. Zimmerman and W. Kruer, Comments Plasma Phys. Controlled Fusion 11, 51 (1975).

${ }^{33}$ M. L. Gittings, in Numerical Methods Symposium, 28-30 April 1992; N. Byrne, T. Betlach, and M. L. Gittings, in Numerical Methods Symposium, 28-30 April 1992. Copies may be ordered from the Defence Nuclear Agency, 56801 Telegraph Road, Alexandria, VA 22310-3398.

${ }^{34}$ P. Colella, SIAM J. Comput. 6, 104 (1985).
${ }^{35}$ R. M. Baltrusaitis, M. L. Gittings, R. P. Weaver, R. F. Benjamin, and J. M. Budzinski, Phys. Fluids 8, 2471 (1996).

${ }^{36}$ R. L. Holmes, G. Dimonte, B. Fryxell, M. L. Gittings, J. W. Grove, M. Schneider, D. Sharp, A. L. Velikovich, R. P. Weaver, and Q. Zhang, J. Fluid Mech. 389, 55 (1999).

${ }^{37}$ R. Tipton, Lawrence Livermore National Laboratory (unpublished); R. T. Barton in Numerical Astrophysics, edited by J. M. Centrella, J. M. LeBlanc, and R. L. Bowers (Jones and Bartlett, Boston, 1985), pp. 482-497.

${ }^{38}$ J. Cantó, G. Tenorio-Tagle, and M. Różyczka, Astron. Astrophys. 192, 287 (1988).

${ }^{39}$ G. Tenorio-Tagle, J. Cantó, and M. Różyczka, Astron. Astrophys. 202, 256 (1988).

${ }^{40}$ L. Spitzer, Jr., The Physics of Fully Ionized Gases, 2nd ed. (Wiley Interscience, New York, 1962), pp. 143-147.

${ }^{41}$ P. E. Dimotakis, J. Fluid Mech. 409, 69 (2000).

${ }^{42}$ H. Li and G. Ben-Dor, AIAA J. 36, 488 (1998).

${ }^{43}$ J. S. Shang, J. Hayes, K. Wurtzler, and W. Strang, AIAA J. 39, 1159 (2001).

${ }^{44}$ J. O. Hinze, Turbulence (McGraw-Hill, New York, 1959), pp. 11-12.

${ }^{45}$ A. Raga, H. Sobral, M. Villagrán-Muniz, R. Navarro-González, and E. Masciadri, Mon. Not. R. Astron. Soc. 324, 206 (2001).

${ }^{46} \mathrm{~S}$. Chandrasekhar, Hydrodynamic and Hydromagnetic Stability (Dover, New York, 1981), p. 481; B. A. Hammel, J. D. Kilkenny, D. Munro, B. A. Remington, H. N. Kornblum, T. S. Perry, D. W. Phillion, and R. J. Wallace, Phys. Plasmas 1, 1662 (1994). 